\title{
Utilization of Echocardiography After Acute Kidney Injury Was Associated with Improved Outcomes in Patients in Intensive Care Unit
}

\author{
Yugang $\mathrm{Hu}^{*}$ \\ Jia Zhou* \\ Quan Cao \\ Hao Wang \\ Yuanting Yang \\ Ye Xiong (D) \\ Qing Zhou
}

Department of Ultrasound Imaging, Renmin Hospital of Wuhan University, Wuhan, 43006I, People's Republic of China

*These authors contributed equally to this work
Correspondence: Qing Zhou

Department of Ultrasound Imaging,

Renmin Hospital of Wuhan University, 99

Ziyang Road, Wuhan, 430060, People's

Republic of China

Tel +86027-8804l91 I

Email qingzhou@whu.edu.cn
Background: We aimed to investigate the association between usage of transthoracic echocardiography (TTE) within 24 hours after acute kidney injury (AKI) and the prognosis of patients in intensive care unit (ICU).

Methods: The Medical Information Mart for Intensive Care III (MIMIC-III) database was used to identify AKI patients with and without TTE administration. The primary outcome was 28-day mortality. Multivariable regression was used to clarify the association between TTE and clinical outcomes and propensity score matching (PSM) and inverse probability of treatment weighting (IPTW) were utilized to validate our findings.

Results: Among 23,945 eligible AKI patients, 3361 patients who received TTE and 3361 who did not conduct TTE had similar propensity scores which were included in this study. After matching, the TTE group had a significantly lower 28-day mortality (OR $0.80,95 \%$ CI $0.72-0.88, \mathrm{P}<0.001)$. Patients in the TTE group received more fluid on day 1 and day 2 and had a more urine volume on day 1 and day 3 , and the reduction in serum creatinine was greater than that in the no TTE group. The mediating effect of creatinine reduction was remarkable for the whole cohort $(\mathrm{P}=0.02$ for the average causal mediation effect).

Conclusion: TTE utilization was associated with decreased risk-adjusted 28-day mortality for AKI patients in ICU and was proportionally mediated through creatinine reduction.

Keywords: echocardiography, acute kidney injury, medical information mart for intensive care, propensity score matching, mortality

\section{Introduction}

Despite great progresses in comprehending the potential pathophysiology and clinical features, acute kidney injury (AKI) is still one of the most frequent complications without any pharmacological treatments and it remains to be a global public health concern with high morbidity, mortality and increased healthcare costs especially in patients in intensive care units (ICU). ${ }^{1-3}$ It is currently acknowledged that timely fluid administration is of great importance in the management of patients with AKI so as to guarantee organ perfusion and therefore traditional management prototype for AKI patients involved highvolume fluid resuscitation. ${ }^{4-6}$ However, more and more evidence demonstrated that excess fluid administration may be in itself harmful to renal recovery or in turn lead to the development of $\mathrm{AKI}^{7-9}$ Thus, a portable and non-invasive instrument to assess the optimal endpoint of fluid resuscitation after AKI may be helpful for clinicians. 
Bedsides transthoracic echocardiography (TTE), a noninvasive and reduplicative hemodynamic management tool, has become cumulatively vital in the administration of fluid balance for critically ill patients and then relevant guidelines have now recommended it as the firstline evaluation modality. ${ }^{10-12}$ However, recent evidence for the utilization of TTE in different populations is controversial. In a large population-based retrospective cohort study, preoperative TTE was not associated with better survival or shorter length of hospital stay for patients following major non-cardiac surgery. ${ }^{13}$ In contrast, in the two latest studies, usage of TTE was proved to be associated with a significant improvement in 28-day mortality in patients with sepsis. ${ }^{14,15}$ Moreover, limited data are available regarding the association between TTE utilization and improved clinical outcomes in patients after AKI, given the superiorities of TTE in the management of fluid balance. Hence, in the current study, we hypothesized that TTE usage after AKI might improve prognosis among patients with AKI because it may facilitate hemodynamic stabilization. We present the following article in accordance with the STARD reporting checklist.

\section{Materials and Methods \\ Data Source}

The data were collected from a large US-based critical care database named Medical Information Mart for Intensive Care III (MIMIC III). ${ }^{16}$ It is a publicly and freely accessible database containing information of 46,520 patients admitted to the ICUs of Beth Israel Deaconess Medical Center in Boston, MA, from June 1, 2001, to October 31, 2012. The database has been approved by the Institutional Review Board (IRB) of the Massachusetts Institute of Technology (MIT). After successfully accomplishing the National Institutes of Health's (NIH) online training course and the Protection of Human Research Participants Examination (certification number 8452818), we had the access to extract data from MIMIC III database. Given that all patients in this database were de-identified, informed consent was waived and data were extracted by structured query language with PostgreSQL 9.6.

\section{Selection of Participants}

Patients in the MIMIC-III who fulfilled the definition of AKI according to KDIGO guideline in the first 48 hours of their ICU admission were eligible for inclusion. ${ }^{17} \mathrm{We}$ excluded patients who were younger than 18 years old or who spent less than 48 hours in the ICU. For patients readmitted to the ICU, only the first ICU admissions were included in this study. Additionally, we further excluded patients who were diagnosed as end-stage renal disease (ESRD). Included patients for whom initial TTE was performed within 24 hours after the diagnosis of AKI was classified as the TTE group, and the rest of the patients comprised the no TTE group (Figure 1).

\section{Variable Extraction}

Baseline characteristics and admission information: age, gender, admission type, weight, first service unit and severity score measured by the sequential organ failure assessment (SOFA) score, the systemic inflammatory response syndrome (SIRS) score, the simplified acute physiology score II (SAPSII) and the elixhauser comorbidity score were calculated as described in previous studies. ${ }^{18-21}$ Comorbidities including hypertension, diabetes, chronic kidney disease (CKD), coronary artery disease (CAD), congestive heart failure (CHF), chronic obstructive pulmonary disease (COPD), liver disease, stroke and

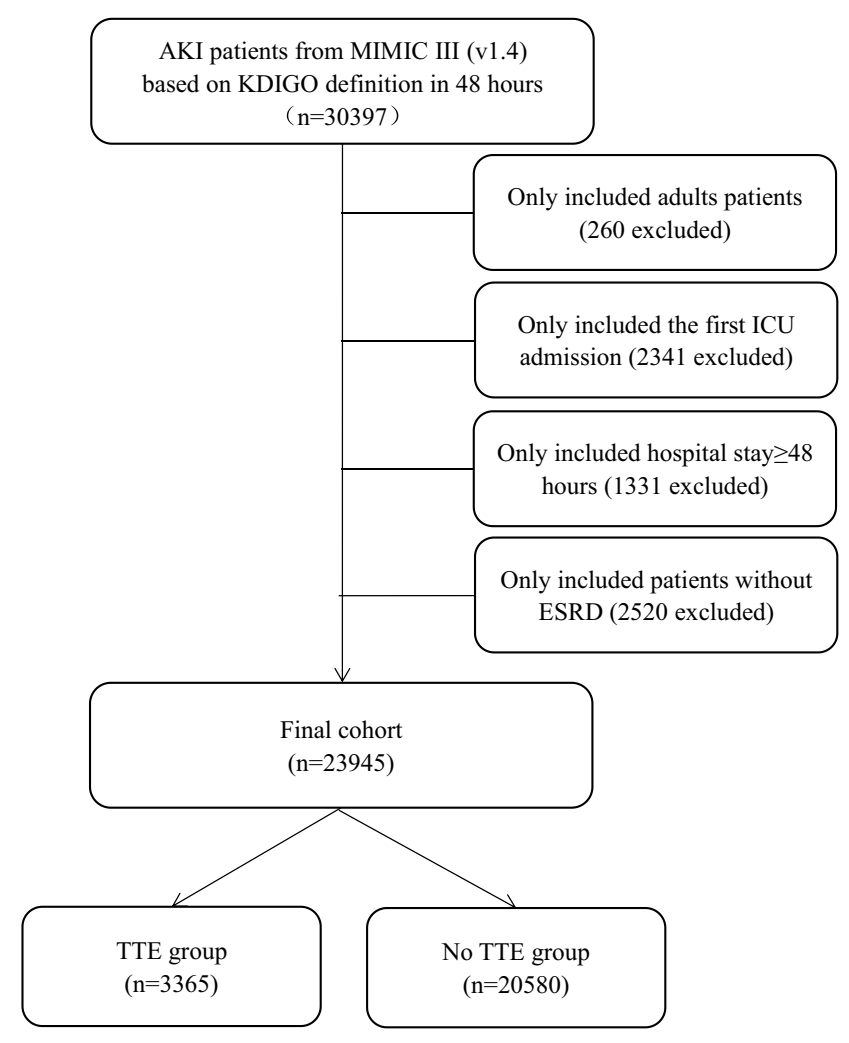

Figure I Study flow diagram in the present study.

Abbreviations: AKI, acute kidney disease; MIMIC III, Medical Information Mart for Intensive Care III; KDIGO, kidney disease: improving global outcomes; ICU, intensive care unit; ESRD, end-stage renal disease; TTE, transthoracic echocardiography. 
malignancy cancer were also collected for analysis based on the recorded International Classification of Diseases-9 (ICD-9) codes in the MIMIC-III database. Use of mechanical ventilation (MV), vasopressors and renal replacement therapy before the diagnosis of AKI were also recorded in this study. Moreover, initial vital signs and laboratory results were also measured during the first 24 hours of ICU admission.

\section{Outcomes}

The primary outcome in this study was 28-day mortality after the day of ICU admission. Secondary outcomes included 1-year morality (death information for discharged patients was obtained from the US Social Security Death Index); the volumes (mL) of intravenous fluid (IVF) and urine output in the first, second and third 24-hour after the diagnosis of $\mathrm{AKI}$; the total number of MV-free, renal replacement therapy (RRT)-free and vasopressor-free days within 28 days after ICU admission; use of diuretics after the diagnosis of AKI; reduction in serum creatinine within 48 hours after the diagnosis of AKI (calculated as the difference between the maximum creatinine level on the day of AKI diagnosis and the first measurement 48 hours after the diagnosis of AKI); and the length of ICU stay and hospital stay.

\section{Statistical Analysis}

Continuous variables were expressed as mean (standard deviation), categorical covariates were reported as number and percentage. We compared the continuous variables using the Wilcoxon signed rank test and Chi-square test was used to compare the categorical covariates. Propensity score matching (PSM) and propensity score-based inverse probability of treatment weighting (IPTW) were also used to adjust the covariates to ensure the robustness of our results. ${ }^{22}$ One-to-one nearest neighbour matching with a calliper width of 0.05 was applied in the current study. An IPTW model was made using the estimated propensity scores as weights. The standardized mean differences (SMDs) were calculated to evaluate the effectiveness of the PSM and IPTW models. Multivariate logistic regression was then conducted on the original cohort, matched cohort and weighted cohort, respectively, to investigate the association between TTE utilization and clinical outcomes. We conducted a series of sensitivity analyses to verify the robustness of the results and the impact of different inference models on the conclusions. We also performed sensitivity analyses focusing only on patients who underwent a single TTE within 24 hours after the diagnosis of AKI to determine if only one echocardiography scan can affect the primary outcome on its own. Causal mediation analysis (CMA) is a tool to distinguish the total effect of a treatment into direct and indirect effects. To explore whether the effect of TTE usage on the primary outcome is proportionally mediated by the reduction in serum creatinine, we used CMA to characterize the causality relationship in this study. All analyses were performed using $\mathrm{R}$ (version 3.3.3) and $\mathrm{p}<0.05$ was considered statistically significant.

\section{Results \\ Patients Characteristics}

A total of 23,945 patients were finally analyzed in this study, with 3365 (14.1\%) patients receiving TTE within 24 hours after the diagnosis of AKI (Figure 1). The baseline characteristics of the TTE and no TTE groups are summarized in Table 1. Before PSM, 16/42 covariates (age, gender, admission type, use of MV and vasopressor, SAPSII score, elixhauser score, CKD, CAD, CHF, respiratory rate, platelet, blood urea nitrogen, $\mathrm{PH}, \mathrm{PO} 2$ and length of days before the diagnosis of AKI) were imbalanced between TTE group and no TTE group. Based on the estimated propensity scores, PSM and IPTW were applied to standardize the differences between the TTE and no TTE groups. As shown in Table 1 and Figure S1, the imbalance between the TTE and no TTE group was significantly reduced and all covariates were comparable between the two groups.

\section{Primary Outcome and Sensitivity Analysis}

The multivariate logistic regression analysis obtained a significant favorable impact of TTE on 28-day mortality (Figure 2), with an adjusted odds ratio (OR) of 0.80 (95\% confidence index, 95\% CI, 0.72-0.88, $\mathrm{P}<0.001)$ and the association remained robust after PSM (OR, 0.60, 95\% CI $0.53-0.68, \mathrm{P}<0.001)$ and IPTW (OR, 0.62, 95\% CI 0.52-$0.69, \mathrm{P}<0.001$ ) (Figure 2). Kaplan-Meier curves also demonstrated that TTE utilization was significantly associated with 28-day mortality before and after PSM (Figure $\underline{\mathrm{S} 2 \mathrm{~A} \text { and } \mathrm{S} 2 \mathrm{~B}) \text {. }}$

We performed a sequence of subgroup analyses to examine patients with different AKI stages and all subgroup analyses showed the similar results (Tables S1-S3). We also performed sensitivity analyses to exclude patients with RRT therapy or with multiple sessions of TTE during 
Table I Comparisons of Baseline Characteristics Between the Original Cohort and Matched Cohort

\begin{tabular}{|c|c|c|c|c|c|c|c|}
\hline \multirow[t]{2}{*}{ Covariate } & \multicolumn{3}{|c|}{ Original Cohort } & \multicolumn{3}{|c|}{ Matched Cohort } & \multirow[t]{2}{*}{ Missing Data (\%) } \\
\hline & No TTE & TTE & SMD & No TTE & TTE & SMD & \\
\hline $\mathrm{N}$ & 20,580 & 3365 & & 3361 & 3361 & & \\
\hline Age, years & $66.8(I 5.8)$ & $68.8(15.0)$ & 0.133 & $69.1(15.8)$ & $68.8(15.0)$ & 0.018 & 0.0 \\
\hline Gender, male, n(\%) & II,760 (57.I) & $1753(52.1)$ & 0.102 & $1748(52.0)$ & $1749(52.0)$ & 0.001 & 0.0 \\
\hline Service unit (MICU) & 7059 (34.3) & $1300(38.6)$ & 0.090 & I33| (39.3) & 1300 (38.7) & 0.006 & 0.0 \\
\hline Weight (kg) & $84.4(24.4)$ & $85.0(25.3)$ & 0.025 & $85.0(26.2)$ & $85.0(25.3)$ & 0.001 & 1.1 \\
\hline $\begin{array}{l}\text { Admission type, } \mathrm{n}(\%) \\
\text { Elective } \\
\text { Emergency } \\
\text { Urgent }\end{array}$ & $\begin{array}{l}4077(19.8) \\
15,922(77.4) \\
58 \mid(2.8)\end{array}$ & $\begin{array}{l}266(7.9) \\
3019(89.7) \\
80(2.4)\end{array}$ & 0.297 & $\begin{array}{l}255(7.6) \\
3011(89.6) \\
95(2.8)\end{array}$ & $\begin{array}{l}266(7.9) \\
3015(89.7) \\
80(2.4)\end{array}$ & 0.030 & 0.0 \\
\hline $\begin{array}{l}\text { Interventions }{ }^{\mathrm{a}}, \mathrm{n}(\%) \\
\text { MV use } \\
\text { Vasopressor use } \\
\text { RRT use }\end{array}$ & $\begin{array}{l}9569(46.5) \\
6239(30.3) \\
295(1.4)\end{array}$ & $\begin{array}{l}939(27.9) \\
647(19.2) \\
60(1.8)\end{array}$ & $\begin{array}{l}0.392 \\
0.259 \\
0.028\end{array}$ & $\begin{array}{l}963(28.7) \\
645(19.2) \\
55(1.6)\end{array}$ & $\begin{array}{l}939(27.9) \\
647(19.3) \\
60(1.8)\end{array}$ & $\begin{array}{l}0.016 \\
0.002 \\
0.011\end{array}$ & $\begin{array}{l}0.0 \\
0.0 \\
0.0\end{array}$ \\
\hline $\begin{array}{l}\text { Severity of illness, n(\%) } \\
\text { SOFA } \\
\text { SIRS } \\
\text { SAPSII }\end{array}$ & $\begin{array}{l}4.7(3.0) \\
2.9(1.0) \\
37.5(13.8)\end{array}$ & $\begin{array}{l}4.9(3.3) \\
2.9(1.0) \\
39.8(13.4)\end{array}$ & $\begin{array}{l}0.070 \\
0.004 \\
0.165\end{array}$ & $\begin{array}{l}4.8(3.2) \\
2.8(1.0) \\
39.7(14.4)\end{array}$ & $\begin{array}{l}4.9(3.3) \\
2.9(1.0) \\
39.9(13.4)\end{array}$ & $\begin{array}{l}0.011 \\
0.013 \\
0.013\end{array}$ & $\begin{array}{l}0.0 \\
0.0 \\
0.0\end{array}$ \\
\hline Elixhauser socre & $14.1(13.1)$ & $17.2(13.3)$ & 0.231 & $17.2(13.4)$ & $17.2(13.3)$ & 0.007 & 0.0 \\
\hline $\begin{array}{l}\text { Comorbidities, } n(\%) \\
\text { Hypertension } \\
\text { Diabetes } \\
\text { CKD } \\
\text { CAD } \\
\text { CHF } \\
\text { COPD } \\
\text { Liver disease } \\
\text { Stroke } \\
\text { Malignancy }\end{array}$ & $\begin{array}{l}9946(48.3) \\
6076(29.5) \\
2376(11.5) \\
6776(32.9) \\
6428(31.2) \\
3006(14.6) \\
1442(7.0) \\
1750(8.5) \\
3586(17.4)\end{array}$ & $\begin{array}{l}1600(47.5) \\
1114(33.1) \\
509(15.1) \\
1287(38.2) \\
1517(45.1) \\
543(16.1) \\
190(5.6) \\
331(9.8) \\
515(15.3)\end{array}$ & $\begin{array}{l}0.016 \\
0.077 \\
0.105 \\
0.111 \\
0.288 \\
0.042 \\
0.056 \\
0.046 \\
0.057\end{array}$ & $\begin{array}{l}1574(46.8) \\
1102(32.8) \\
532(15.8) \\
1289(38.4) \\
1520(45.2) \\
559(16.6) \\
194(5.8) \\
354(10.5) \\
514(15.3)\end{array}$ & $\begin{array}{l}1597(47.5) \\
1112(33.1) \\
509(15.1) \\
1283(38.2) \\
1514(45.0) \\
543(16.2) \\
189(5.6) \\
330(9.8) \\
515(15.3)\end{array}$ & $\begin{array}{l}0.014 \\
0.006 \\
0.019 \\
0.004 \\
0.004 \\
0.013 \\
0.006 \\
0.024 \\
0.001\end{array}$ & $\begin{array}{l}0.0 \\
0.0 \\
0.0 \\
0.0 \\
0.0 \\
0.0 \\
0.0 \\
0.0 \\
0.0\end{array}$ \\
\hline $\begin{array}{l}\text { Vital signs } \\
\text { MAP, mmHg } \\
\text { Heart rate, bpm } \\
\text { Respiratory rate, bpm } \\
\text { Temperature }\left({ }^{\circ} \mathrm{C}\right)\end{array}$ & $\begin{array}{l}106.0(27.7) \\
71.6(14.5) \\
12.1(3.8) \\
36.0(0.8)\end{array}$ & $\begin{array}{l}104.8(28.5) \\
71.0(16.0) \\
12.8(3.8) \\
36.0(0.9)\end{array}$ & $\begin{array}{l}0.042 \\
0.036 \\
0.196 \\
0.013\end{array}$ & $\begin{array}{l}105.0(26.4) \\
71.0(15.1) \\
12.9(3.9) \\
36.1(0.8)\end{array}$ & $\begin{array}{l}104.8(28.5) \\
71.1(16.0) \\
12.8(3.8) \\
36.0(0.9)\end{array}$ & $\begin{array}{l}0.005 \\
0.007 \\
0.014 \\
0.014\end{array}$ & $\begin{array}{l}0.5 \\
0.4 \\
0.5 \\
2.9\end{array}$ \\
\hline $\begin{array}{l}\text { Laboratory results } \\
\text { WBC, }\left(\times 10^{9} / \mathrm{L}\right) \\
\text { HGB, g/dL } \\
\text { PLT, }\left(\times 10^{9} / \mathrm{L}\right) \\
\text { Bilirubin, }(\mathrm{mmol} / \mathrm{L}) \\
\text { Albumin, } \mathrm{g} / \mathrm{dL} \\
\text { Aniongap, } \mathrm{mEq} / \mathrm{L} \\
\text { Bicarbonate, } \mathrm{mEq} / \mathrm{L} \\
\text { BUN, } \mathrm{mg} / \mathrm{dL} \\
\text { Creatinine, } \mathrm{mg} / \mathrm{dL}\end{array}$ & $\begin{array}{l}11.7(7.3) \\
10.9(2.1) \\
231.3(127.9) \\
1.6(3.5) \\
3.1(0.6) \\
14.0(3.9) \\
24.9(4.8) \\
26.8(20.1) \\
1.3(1.1)\end{array}$ & $\begin{array}{l}\text { II.8 (I0.5) } \\
\text { II.0 (2.I) } \\
238.8(128.0) \\
1.5(3.5) \\
3.1(0.6) \\
13.9(4.0) \\
24.8(5.1) \\
29.4(20.8) \\
1.4(1.1)\end{array}$ & $\begin{array}{l}0.017 \\
0.074 \\
0.184 \\
0.041 \\
0.013 \\
0.024 \\
0.020 \\
0.131 \\
0.071\end{array}$ & $\begin{array}{l}11.7(7.5) \\
11.0(2.0) \\
242.1(130.6) \\
1.5(2.9) \\
3.1(0.6) \\
13.9(3.7) \\
24.9(5.1) \\
29.9(22.1) \\
1.4(1.3)\end{array}$ & $\begin{array}{l}\text { II.8 (10.5) } \\
\text { II.0 (2.I) } \\
238.8(128.0) \\
1.5(3.5) \\
3.1(0.6) \\
13.9(4.0) \\
24.8(5.1) \\
29.4(20.7) \\
1.4(1.1)\end{array}$ & $\begin{array}{l}0.014 \\
0.003 \\
0.025 \\
0.014 \\
0.014 \\
0.001 \\
0.010 \\
0.022 \\
0.033\end{array}$ & $\begin{array}{l}0.1 \\
0.1 \\
0.1 \\
29.0 \\
31.3 \\
0.1 \\
0.1 \\
0.1 \\
0.1\end{array}$ \\
\hline
\end{tabular}

(Continued) 
Table I (Continued).

\begin{tabular}{|c|c|c|c|c|c|c|c|}
\hline \multirow[t]{2}{*}{ Covariate } & \multicolumn{3}{|c|}{ Original Cohort } & \multicolumn{3}{|c|}{ Matched Cohort } & \multirow[t]{2}{*}{ Missing Data (\%) } \\
\hline & No TTE & TTE & SMD & No TTE & TTE & SMD & \\
\hline Lactate, $\mathrm{mmol} / \mathrm{L}$ & $2.2(1.6)$ & $2.2(1.8)$ & 0.037 & $2.2(1.6)$ & $2.2(1.8)$ & 0.002 & 24.2 \\
\hline Potassium, $\mathrm{mmol} / \mathrm{L}$ & $3.7(0.5)$ & $3.8(0.6)$ & 0.063 & $3.8(0.6)$ & $3.8(0.6)$ & 0.007 & 0.2 \\
\hline Sodium, $\mathrm{mmol} / \mathrm{L}$ & I $36.3(5.1)$ & I $36.3(4.8)$ & 0.009 & I36.2 (5.7) & I $36.3(4.8)$ & 0.002 & 0.2 \\
\hline $\mathrm{PH}$ & $7.37(0.08)$ & $7.36(0.08)$ & 0.143 & $7.36(0.08)$ & $7.36(0.08)$ & 0.011 & 35.7 \\
\hline $\mathrm{PO} 2, \mathrm{mmHg}$ & $235.6(118.9)$ & $204.0(103.3)$ & 0.284 & 200.2 (I0I.I) & $204.2(103.2)$ & 0.039 & 39.6 \\
\hline $\mathrm{PCO} 2, \mathrm{mmHg}$ & $43.8(10.6)$ & $44.2(11.2)$ & 0.032 & $44.2(11.3)$ & $44.2(11.2)$ & 0.004 & 39.6 \\
\hline Days before AKI & $1.0(0.8)$ & $0.6(0.8)$ & 0.478 & $0.7(0.8)$ & $0.6(0.8)$ & 0.019 & 0.0 \\
\hline AKI stages & & & 0.091 & & & 0.027 & 0.0 \\
\hline Stage I & $6292(30.6)$ & $903(26.8)$ & & $936(27.8)$ & $903(26.9)$ & & \\
\hline Stage II & $|0,84|(52.7)$ & $|82|(54.1)$ & & $1776(52.8)$ & $1819(54.1)$ & & \\
\hline Stage III & $3447(16.7)$ & $641(19.0)$ & & $649(19.3)$ & $639(19.0)$ & & \\
\hline
\end{tabular}

Notes: For all continuous covariates, the mean values and standard deviations are reported. ${ }^{\mathrm{a}} \mathrm{All}$ of interventions were conducted before the diagnosis of acute kidney disease.

Abbreviations: TTE, transthoracic echocardiography; SMD, standardized mean difference; MICU, medical intensive care; MV, mechanical ventilation; RRT, renal replacement therapy; SOFA, sequential organ failure assessment; SIRS, systemic inflammatory response syndrome; SAPS II, Simplified Acute Physiology Score II; CKD, chronic kidney disease; $C A D$, coronary artery disease; $C H F$, congestive heart failure; COPD, chronic obstructive pulmonary disease; MAP, mean arterial pressure; WBC, white blood cell; HGB, hemoglobin; PLT, platelet; BUN, blood urea nitrogen; PO2, partial pressure of oxygen; PCO2, partial pressure of carbon dioxide; AKI, acute kidney injury.

ICU stay and produced the same findings (Tables S4 and $\underline{\mathrm{S} 5}$ ). Moreover, the different duration of the diagnosis of AKI did not alter the association between TTE utilization and better 28-survival rate (Table S6).

\section{Secondary Outcomes Studies with Propensity Score Matching}

Patients in the TTE group had a better 1-year survival rate compared with patients in the no TTE group (33.1\% vs $35.7 \%, \mathrm{P}=0.022)$. We conducted a series of secondary outcomes to evaluate potential factors that might be obligated to the conducive effects of the usage of TTE. Compared with patients in the no TTE group, patients in the TTE group had higher volume of IVF on the first day ( 0.9 vs $0.7 \mathrm{~L}, \mathrm{P}<0.001)$ and the second day $(0.3$ vs $0.2 \mathrm{~L}$, $\mathrm{P}=0.007$ ) after the diagnosis of AKI. However, there was no difference on the third day. Patients in the TTE group had higher volume of urine output on the first day $(1.5 \mathrm{vs} 1.4 \mathrm{~L}$, $\mathrm{P}<0.001)$ and the third day $(0.8$ vs $0.7 \mathrm{~L}, \mathrm{P}=0.011)$ after the diagnosis of AKI, but there was no difference on the second

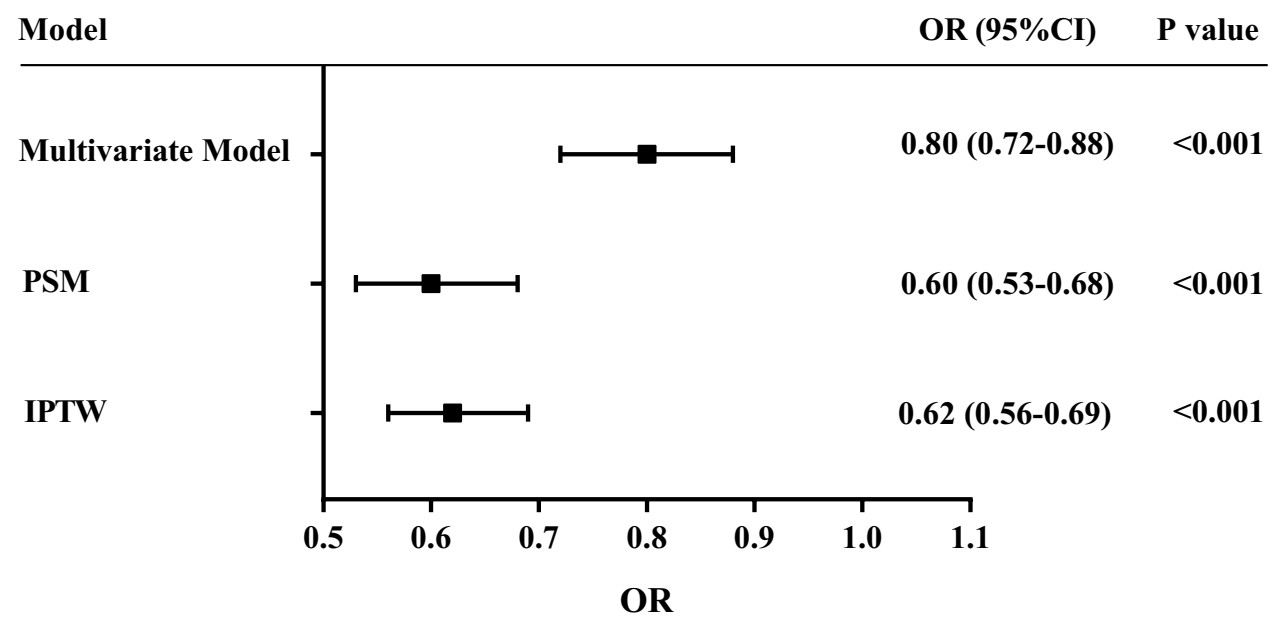

Figure 2 Association between transthoracic echocardiography (TTE) and 28-day mortality. The odds ratios (OR) and $95 \%$ confidence intervals (95\% Cl, error bars) in both cohorts were calculated dependent on the method of covariate adjustment.

Abbreviations: PSM, propensity score matching; IPTW, inverse probability of treatment weighing. 
day. The TTE group had a significantly higher duration of vasopressor use (vasopressor-free days in 28 days of 27.1 vs 26.8, $\mathrm{P}<0.001$ ) and mechanical ventilation (ventilation-free days in 28 days of 25.6 vs $26.0, \mathrm{P}=0.001$ ). The duration of RRT therapy did not significantly differ between two groups. The use of diuretic ( $54.1 \%$ vs.44.2\%, $\mathrm{P}<0.001)$ was significantly higher for the TTE patients. With respect to serum creatinine, we found that the reduction in serum creatinine between day 1 and day 3 after the diagnosis of AKI was higher in the TTE group than in the no TTE group ( 0.40 vs $0.34 \mathrm{mg} / \mathrm{dL}, \mathrm{P}<0.001)$. Moreover, TTE group had longer ICU stay than patients in the no TTE group, but was comparable on the length of hospital stay. All of the secondary outcomes are summarized in Table 2.

CMA showed that the reduction in serum creatinine mediated $3.6 \%(95 \%$ CI $0.7 \%-7.0 \% ; \mathrm{P}=0.02)$ of the beneficial effect of TTE utilization $(\mathrm{P}=0.02$ for average causal mediation effects) in terms of 28-day mortality in AKI patients (Figure 3).

\section{Discussion}

In the current study, we found that usage of TTE within 24 hours after AKI was associated with decreased 28-day mortality as well as improved 1-year survival, and this association became robust in the sensitivity analyses. Moreover, TTE utilization was proportionally mediated through creatinine reduction. To the best of our knowledge, this was the first study to investigate the impact of TTE utilization on the prognosis of AKI patients.

Despite the different diagnostic criteria have been used formerly, making it out of the question to draw precise conclusions on the prevalence of this syndrome, AKI is incontrovertibly a global public health problem affecting about 13.3 million patients per year. ${ }^{23}$ Furthermore, although great progresses in the management of AKI patients together with renal replacement therapy and multi-organ support therapy, personalized pharmacological therapy, AKI still associated with high mortality - nearly 1.7 million deaths per year and high risk of ESRD. ${ }^{24}$

Correction of intravascular hypovolemia is critical for the management of patients with AKI; thus, the conventional tactic for patients with developed AKI or at high risk of AKI often involves the utilization of fluids because of the potential untreated hypovolemia. ${ }^{25}$ However, there is growing evidence demonstrated that superfluous fluid resuscitation over correction of hypovolemia could in turn result in adverse clinical outcomes, including the development and even the progression of AKI. ${ }^{5,26}$ Based on the data from Sepsis Occurrence in Acutely ill Patients (SOAP) study, a large multicenter observational cohort study to which 198 ICUs from 24 European countries contributed, Payen et al concluded that a positive fluid

Table 2 Clinical Outcomes Analysis with Propensity Score Matched Cohorts

\begin{tabular}{|c|c|c|c|c|c|}
\hline Outcomes & No TTE & TTE & Effect Size & $P$ value & Missing Data (\%) \\
\hline \multicolumn{6}{|l|}{ Primary outcome } \\
\hline 28-day mortality & $766(22.8)$ & $508(15.1)$ & 0.197 & $<0.001$ & 0.0 \\
\hline \multicolumn{6}{|l|}{ Secondary outcomes } \\
\hline I-year mortality & $1200(35.7)$ & $1111(33.1)$ & 0.056 & 0.022 & 0.0 \\
\hline Ventilation-free days in 28 days & $26.0(4.8)$ & $25.6(5.4)$ & 0.069 & 0.005 & 0.0 \\
\hline Vasopressor-free days in 28 days & $27.1(2.6)$ & $26.8(3.1)$ & 0.121 & $<0.001$ & 0.0 \\
\hline RRT-free days in 28 days & $27.9(1.1)$ & $27.8(1.4)$ & 0.041 & 0.090 & 0.0 \\
\hline Usage of diuretic & $1486(44.2)$ & $1818(54.1)$ & 0.199 & $<0.001$ & 0.0 \\
\hline Volume of IVF on day I $(\mathrm{mL})^{\mathrm{a}}$ & $708.8(1467.6)$ & $907.9(1724.0)$ & 0.120 & $<0.001$ & 19.7 \\
\hline Volume of IVF on day $2(\mathrm{~mL})^{\mathrm{b}}$ & $239.5(706.0)$ & $290.5(875.2)$ & 0.065 & 0.007 & 44.5 \\
\hline Volume of IVF on day $3(\mathrm{~mL})^{\mathrm{b}}$ & $120.0(474.3)$ & $125.3(457.8)$ & 0.011 & 0.753 & 59.3 \\
\hline Urine volume on day $\mathrm{I}(\mathrm{mL})^{\mathrm{a}}$ & |359.4 (| ||7.6) & $1492.9(|| \mid 12.4)$ & 0.120 & $<0.001$ & 10.3 \\
\hline Urine volume on day $2(\mathrm{~mL})^{\mathrm{b}}$ & $731.8(951.4)$ & $746.8(967.6)$ & 0.016 & 0.547 & 17.7 \\
\hline Urine volume on day $3(\mathrm{~mL})^{\mathrm{b}}$ & $740.8(1010.6)$ & $830.7(1089.3)$ & 0.086 & 0.011 & 39.2 \\
\hline Creatinine reduction in 48 hours $^{c}$ & $0.34(0.6 \mathrm{I})$ & $0.40(0.55)$ & 0.110 & $<0.001$ & 9.2 \\
\hline Length of ICU stay & $5.31(6.52)$ & $5.93(7.27)$ & 0.089 & $<0.001$ & 0.0 \\
\hline Length of hospital stay & $11.99(10.43)$ & $12.05(10.89)$ & 0.006 & 0.803 & 0.0 \\
\hline
\end{tabular}

Notes: ${ }^{a}$ Volume of IVF and urine volume on day I mean the total of IV fluid or urine volume in first 24 hours after the diagnosis of AKI. ${ }^{b}$ Volume of IVF and urine volume on day 2 or 3 mean the total of IV fluid or urine volume in second or third 24 hours after the diagnosis of AKI and patients who were dead or discharged from the hospital before day 2 or day 3 were excluded. 'Patients who were dead or discharged from the hospital within 48 hours after AKI diagnosed were also excluded.

Abbreviations: RRT, renal replacement therapy; IVF, intravenous fluid; ICU, intensive care unit. 


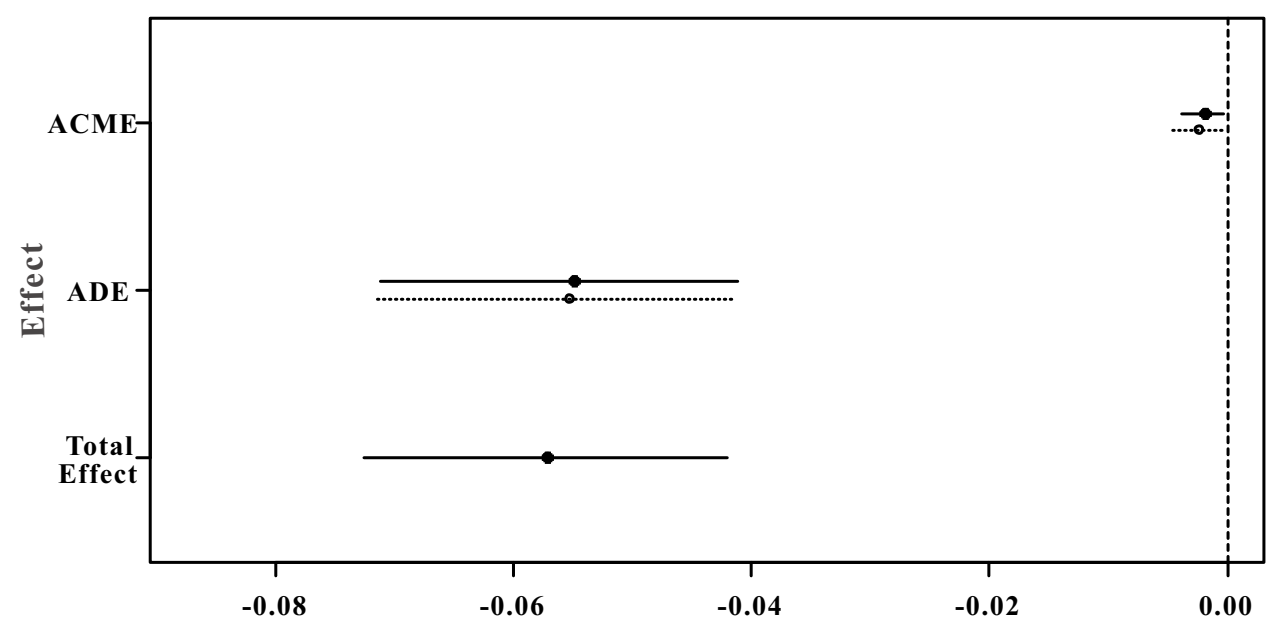

Causal mediation analysis for serum creatinine reduction

Figure 3 Causal mediation analysis for serum creatinine reduction. The solid line represents the transthoracic echocardiography (TTE) group, and the dashed line represents the no TTE group.

Abbreviations: ACME, average causal mediation effects; ADE, average direct effects.

balance was an important risk factor associated with increased 60-day mortality in critically ill patients with acute renal failure. ${ }^{27}$ Similarly, a recent prospective multicenter study of 1734 ICU patients demonstrated that maximum fluid overload was associated with increased inhospital mortality $(\mathrm{OR}=1.044,95 \%$ CI $1.023-1.065)$ even after adjusting for severity of illness and AKI status. ${ }^{28}$ Therefore, exactly evaluating and administering the volume status and determining the optimal endpoint of fluid resuscitation can effectively improve clinical outcomes in critically ill patients.

Several strategies, including mean arterial pressure (MAP), central venous pressure (CVP), pulmonary artery occlusion pressure and so on, have been proposed for hemodynamic monitoring in previous studies. ${ }^{29,30}$ MAP was often considered to serve as an important variable for assessing organ perfusion and hemodynamic stability was even defined by $\mathrm{MAP} \geq 65 \mathrm{mmHg}$ for 2 consecutive hours in previous study. ${ }^{31}$ However, kidney perfusion depends on the efferent arteriole which is often much lower than the systemic MAP. ${ }^{32}$ CVP remains the most regularly used index to guide fluid resuscitation for ICU patients and a target value of $8-12 \mathrm{mmHg}$ becomes "standard" for clinicians to assess the respond of fluid. ${ }^{33,34}$ However, in patients without indices of hypoperfusion, this target value is not suggested as it could lead to excessive fluid administration. ${ }^{35}$ Above all, TTE may be more reliable and safe technique in that it can provide realtime information of volume status and cardiac function during fluid administration to prevent fluid overload. ${ }^{10,11}$ However, limited data are available in the literature for how to echocardiography in AKI patients. Sapp et al retrospectively enrolled 2413 trauma patients who admitted to level I trauma centra's ICU and revealed that transesophageal echocardiography can provide valuable information to reserve RRT for worse AKIs and decreasing hospital costs. $^{36}$

In the current study, the SOFA score, SAPSII score, serum creatinine and incidence of most comorbid conditions were much higher in the TTE group than in the no TTE group. Patients in the TTE group had higher proportion of advanced AKI stage while the MAP was lower than in the no TTE group. Despite all of those results pointing to a severe group of patients, prominent lower in-hospital mortality as well as 1-year mortality were observed in the TTE group patients than in the no TTE group patients independent of other common risk factors. Thus, due to directly and sequentially evaluating the cardiac output, function and preload, TTE could better characterize the hemodynamic disorders and their response to therapy in ICU patients with AKI. Moreover, we recommend the wide application of TTE in most hospitals for a quick, reduplicative, and non-invasive discrimination between hypervolemic and hypovolemic, as this instrument is based on easily accessible technique and clinicians could acquire it quickly with simple training. For critical ill patients with an unknown volume status in a primary hospital and no hardware conditions for other upscale 
methods for determining and monitoring the fluid status, TTE could provide an important reference to help clinicians discriminate hypervolemic and hypovolemic and determine the optimal endpoint of fluid input.

Previous studies have demonstrated that early timing of recovery from AKI is associated with improved clinical outcomes. ${ }^{37,38}$ In this study, we assumed that TTE-related triggers including fluid therapy could reduce the elevated serum creatinine levels and further improve outcomes. The volume of IVFs on day 1 and day 2 and the reduction in serum creatinine were higher in the TTE group than in the no TTE group. We used TTE as the treatment and the creatinine reduction in 48 hours as a mediator variable and found that the effect of TTE on 28-day mortality was proportionally mediated by the reduction in serum creatinine.

Several limitations should be considered in the current study. First of all, this was a retrospective study based on a single-center electronic public database, which may result in limited generalizability. Secondly, the definition of AKI based on the first 48 hours after ICU admission, thus some patients with AKI may not include in this study. Thirdly, some important clinical variables were missing in this database, including detailed measurements from TTE reports. Finally, the causal relationship between TTE and prognosis of patients with AKI was not entirely explored as TTE-related triggers are complex in clinical practice. A randomized study comparing the effect of TTE and no TTE is needed in the future.

\section{Conclusions}

In this study, we firstly demonstrated that usage of TTE within 24 hours after AKI occurrence resulted in subsequent active changes in management and more importantly, TTE was associated with improved 28-day survival as well as 1-year survival in AKI patients in ICU and was proportionally mediated through creatinine reduction.

\section{Statement of Ethics}

The study has been approved by the Institutional Review Board (IRB) of the Massachusetts Institute of Technology (MIT). After successfully accomplishing the National Institutes of Health's (NIH) online training course and the Protection of Human Research Participants Examination (certification number 8452818), we had the access to extract data from MIMIC III database. Given that all patients in this database were de-identified, informed consent was waived.

\section{Author Contributions}

All authors made substantial contributions to conception and design, acquisition of data, or analysis and interpretation of data; took part in drafting the article or revising it critically for important intellectual content; agreed to submit to the current journal; gave final approval of the version to be published; and agree to be accountable for all aspects of the work.

\section{Funding}

This study was supported by the Nature and Science Foundation of China (No. 81971624) and Wuhan Science and Technology Bureau Application Foundation Frontier (2019020701011477).

\section{Disclosure}

The authors declared that there is no conflict of interest.

\section{References}

1. Ronco C, Bellomo R, Kellum JA. Acute kidney injury. Lancet. 2019;394:1949-1964. doi:10.1016/S0140-6736(19)32563-2

2. Hoste E, Kellum JA, Selby NM, et al. Global epidemiology and outcomes of acute kidney injury. Nat Rev Nephrol. 2018;14:607-625. doi:10.1038/s41581-018-0052-0

3. Negi S, Koreeda D, Kobayashi S, et al. Acute kidney injury: epidemiology, outcomes, complications, and therapeutic strategies. Semin Dial. 2018;31:519-527. doi:10.1111/sdi.12705

4. Martinez-Garcia JJ, Leon-Sicairos NM, Canizalez-Roman A, GarciaArellano BA. Fluid balance and acute kidney injury in septic shock. Bol Med Hosp Infant Mex. 2017;74:282-288. doi:10.1016/j. bmhimx.2017.02.002

5. Finfer S, Myburgh J, Bellomo R. Intravenous fluid therapy in critically ill adults. Nat Rev Nephrol. 2018;14:541-557. doi:10.1038/ s41581-018-0044-0

6. Ostermann M, Liu K, Kashani K. Fluid management in acute kidney injury. Chest. 2019;156:594-603. doi:10.1016/j.chest.2019.04.004

7. Stein A, de Souza LV, Belettini CR, et al. Fluid overload and changes in serum creatinine after cardiac surgery: predictors of mortality and longer intensive care stay. A prospective cohort study. Crit Care. 2012;16:R99. doi:10.1186/cc11368

8. Liu Y, Xue FS, Liu YY, Yang GZ. Assessing association between fluid balance and acute kidney injury after cardiac surgery. $J$ Crit Care. 2018;45:249. doi:10.1016/j.jcrc.2017.12.021

9. Hatton GE, Du RE, Wei S, et al. Positive fluid balance and association with post-traumatic acute kidney injury. $J$ Am Coll Surg. 2020;230:190-199. doi:10.1016/j.jamcollsurg.2019.10.009

10. Johnson A, Mohajer-Esfahani M. Exploring hemodynamics: a review of current and emerging noninvasive monitoring techniques. Crit Care Nurs Clin North Am. 2014;26:357-375. doi:10.1016/j.ccell.2014.05.001

11. Cecconi M, De Backer D, Antonelli M, et al. Consensus on circulatory shock and hemodynamic monitoring. Task force of the European Society of Intensive Care Medicine. Intensive Care Med. 2014;40:1795-1815. doi:10.1007/s00134-014-3525-z

12. Levitov A, Frankel HL, Blaivas M, et al. Guidelines for the appropriate use of bedside general and cardiac ultrasonography in the evaluation of critically ill patients-part II: cardiac ultrasonography. Crit Care Med. 2016;44:1206-1227. doi:10.1097/CCM.00000000 00001847 
13. Wijeysundera DN, Beattie WS, Karkouti K, Neuman MD, Austin PC, Laupacis A. Association of echocardiography before major elective non-cardiac surgery with postoperative survival and length of hospital stay: population based cohort study. BMJ. 2011;342:d3695. doi:10.1136/bmj.d3695

14. Feng M, McSparron JI, Kien DT, et al. Transthoracic echocardiography and mortality in sepsis: analysis of the MIMIC-III database. Intensive Care Med. 2018;44:884-892. doi:10.1007/s00134-0185208-7

15. Lan P, Wang TT, Li HY, et al. Utilization of echocardiography during septic shock was associated with a decreased 28-day mortality: a propensity score-matched analysis of the MIMIC-III database. Ann Transl Med. 2019;7:662. doi:10.21037/atm.2019.10.79

16. Johnson AE, Pollard TJ, Shen L, et al. MIMIC-III, a freely accessible critical care database. Sci Data. 2016;3:160035. doi:10.1038/ sdata. 2016.35

17. Kellum JA, Lameire N. Diagnosis, evaluation, and management of acute kidney injury: a KDIGO summary (Part 1). Crit Care. 2013;17:204. doi:10.1186/cc11454

18. Le Gall JR, Loirat P, Alperovitch A, et al. A simplified acute physiology score for ICU patients. Crit Care Med. 1984;12:975-977. doi:10.1097/00003246-198411000-00012

19. Vincent JL, Moreno R, Takala J, et al. The SOFA (Sepsis-related Organ Failure Assessment) score to describe organ dysfunction/failure. On behalf of the Working Group on Sepsis-Related Problems of the European Society of Intensive Care Medicine. Intensive Care Med. 1996;22:707-710. doi:10.1007/BF01709751

20. van Walraven C, Austin PC, Jennings A, Quan H, Forster AJ. A modification of the Elixhauser comorbidity measures into a point system for hospital death using administrative data. Med Care. 2009;47:626-633. doi:10.1097/MLR.0b013e31819432e5

21. Rass V, Gaasch M, Kofler M, et al. Systemic inflammatory response syndrome as predictor of poor outcome in nontraumatic subarachnoid hemorrhage patients. Crit Care Med. 2018;46:e1152-9. doi:10.1097/ CCM.0000000000003429

22. Graffeo N, Latouche A, Le Tourneau C, Chevret S. ipcwswitch: an $\mathrm{R}$ package for inverse probability of censoring weighting with an application to switches in clinical trials. Comput Biol Med. 2019;111:103339. doi:10.1016/j.compbiomed.2019.103339

23. Mehta RL, Cerda J, Burdmann EA, et al. International Society of Nephrology's 0by25 initiative for acute kidney injury (zero preventable deaths by 2025): a human rights case for nephrology. Lancet. 2015;385:2616-2643. doi:10.1016/S0140-6736(15)60126-X

24. Grams ME, Sang Y, Coresh J, et al. Candidate surrogate end points for ESRD after AKI. J Am Soc Nephrol. 2016;27:2851-2859. doi:10.1681/ASN.2015070829

25. Rhodes A, Evans LE, Alhazzani W, et al. Surviving sepsis campaign: international guidelines for management of sepsis and septic shock: 2016. Intensive Care Med. 2017;43:304-377.
26. Martensson J, Bellomo R. Does fluid management affect the occurrence of acute kidney injury? Curr Opin Anaesthesiol. 2017;30:84-91. doi:10.1097/ACO.0000000000000407

27. Payen D, de Pont AC, Sakr Y, Spies C, Reinhart K, Vincent JL. A positive fluid balance is associated with a worse outcome in patients with acute renal failure. Crit Care. 2008;12:R74. doi:10.1186/cc6916

28. Garzotto F, Ostermann M, Martin-Langerwerf D, et al. The Dose Response Multicentre Investigation on Fluid Assessment (DoReMIFA) in critically ill patients. Crit Care. 2016;20:196. doi:10.1186/s13054-016-1355-9

29. Marik PE, Cavallazzi R. Does the central venous pressure predict fluid responsiveness? An updated meta-analysis and a plea for some common sense. Crit Care Med. 2013;41:1774-1781. doi:10.1097/ CCM.0b013e31828a25fd

30. Eskesen TG, Wetterslev M, Perner A. Systematic review including re-analyses of 1148 individual data sets of central venous pressure as a predictor of fluid responsiveness. Intensive Care Med. 2016;42:324-332. doi:10.1007/s00134-015-4168-4

31. Adams C, Tucker C, Allen B, et al. Disparities in hemodynamic resuscitation of the obese critically ill septic shock patient. J Crit Care. 2017;37:219-223. doi:10.1016/j.jcrc.2016.10.004

32. Kato R, Pinsky MR. Personalizing blood pressure management in septic shock. Ann Intensive Care. 2015;5:41. doi:10.1186/s13613015-0085-5

33. Rivers E, Nguyen B, Havstad S, et al. Early goal-directed therapy in the treatment of severe sepsis and septic shock. $N$ Engl J Med. 2001;345:1368-1377. doi:10.1056/NEJMoa010307

34. De Backer D, Vincent JL. Should we measure the central venous pressure to guide fluid management? Ten answers to 10 questions. Crit Care. 2018;22:43. doi:10.1186/s13054-018-1959-3

35. Semler MW, Wheeler AP, Thompson BT, Bernard GR, Wiedemann HP, Rice TW. Impact of initial central venous pressure on outcomes of conservative versus liberal fluid management in acute respiratory distress syndrome. Crit Care Med. 2016;44:782-789. doi:10.1097/CCM.0000000000001555

36. Sapp A, Drahos A, Lashley M, Christie A, Christie DB. The impact of hemodynamic transesophageal echocardiography on acute kidney injury management and use of continuous renal replacement therapy in trauma. Am Surg. 2020;86:190-194. doi:10.1177/ 000313482008600326

37. Kellum JA, Sileanu FE, Bihorac A, Hoste EA, Chawla LS. Recovery after acute kidney injury. Am $J$ Respir Crit Care Med. 2017;195:784-791. doi:10.1164/rccm.201604-07990C

38. Siew ED, Abdel-Kader K, Perkins AM, et al. Timing of recovery from moderate to severe AKI and the risk for future loss of kidney function. Am J Kidney Dis. 2020;75:204-213. doi:10.1053/j. ajkd.2019.05.031
International Journal of General Medicine

\section{Publish your work in this journal}

The International Journal of General Medicine is an international, peer-reviewed open-access journal that focuses on general and internal medicine, pathogenesis, epidemiology, diagnosis, monitoring and treatment protocols. The journal is characterized by the rapid reporting of reviews, original research and clinical studies across all disease areas. The manuscript management system is completely online and includes a very quick and fair peer-review system, which is all easy to use. Visit http://www.dovepress.com/ testimonials.php to read real quotes from published authors. 